\title{
Novel Spiro-Configured PET Chromophores Incorporating \\ 4,5-Diazafluorene Moiety as an Electron Acceptor
}

\author{
Ken-Tsung Wong, ${ }^{*}$ Hsiao-Fan Chen, and Fu-Chuan Fang \\ Department of Chemistry, National Taiwan University, Taipei 106, Taiwan \\ kenwong@ntu.edu.tw
}

Received May 18, 2006

\section{ABSTRACT}

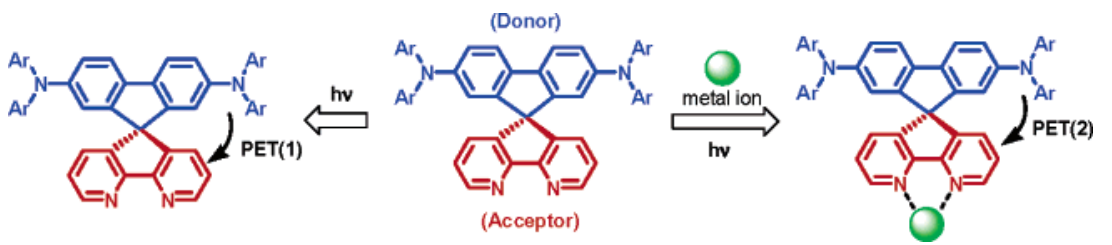

We have synthesized novel spiro-bridged chromophores incorporating a 4,5-diazafluorene unit as the electron acceptor (A) and aryl amino groups as donor (D) moieties. The orthogonal D-A configuration limits ground-state D-A interactions but allows efficient PET reactions. The PET efficiency was modulated by the electronic characteristics of the donor and by the D-A distance. Preliminary studies showed that the PET process can be manipulated through metal ion complexation of the 4,5-diazafluorene moiety.

The concept of photoinduced electron transfer (PET) is applied widely in the design of functional organic molecules incorporating electron donor (D) and acceptor (A) chromophores. Many molecular devices that function through PET mechanisms have been developed, including molecular rectifiers, ${ }^{1}$ electrochemical sensors, ${ }^{2}$ switches,${ }^{3}$ photovoltaic cells, ${ }^{4}$ and nonlinear optical materials. ${ }^{5}$ A major challenge

(1) (a) Molecular Electronics; Jortner, J., Ratner, M., Eds.; Blackwell Science: Oxford, UK, 1997. (b) Joachim, C.; Gimzewski, J. K.; Aviram, A. Nature 2000, 408, 541. (c) Scheib, S.; Cava, M. P.; Baldwin, J. W. Metzger, R. M. J. Org. Chem. 1998, 63, 1198. (e) Majumder, C.; Mizuseki, H.; Kawazoe, Y. J. Phys. Chem. A 2001, 105, 9454

(2) (a) Pond, S. J. K.; Tsutsumi, O.; Rumi, M.; Kwon, O.; Zojer, E.; Brédas, J.-L.; Marder, S. R.; Perry, J. W. J. Am. Chem. Soc. 2004, 126 , 9291. (b) Kim, H. M.; Jeong, M.-Y.; Ahn, H. C.; Jeon, S.-J.; Cho, B. R. J. Org. Chem. 2004, 69, 5749. (c) Miura, T.; Urano, Y.; Tanaka, K.; Nagano, T.; Ohkubo, K.; Fukuzumi, S. J. Am. Chem. Soc. 2003, 125, 8666. (d) Gabe, Y.; Urano, Y.; Kikuchi, K.; Kojima, H.; Nagano, T. J. Am. Chem. Soc. 2004, 126, 3357. (e) Thiagarajan, V.; Ramamurthy, P.; Thirumalai, D.; Ramakrishnan, V. T. Org. Lett. 2005, 7, 657.

(3) (a) Molecular Switches; Feringa, B. L., Ed.: Wiley-VCH: New York, 2001. (b) Straight, S. D.; Andreasson, J.; Kodis, G.; Moore, A. L.; Moore, T. A.; Gust, D. J. Am. Chem. Soc. 2005, 127, 2717. (c) Belser, P.; De Cola, L.; Hartl, F.; Adamo, V.; Bozic, B.; Iyer, Y. C. V. M.; Jukes, R. T. F.; Kühni, J.; Querol, M.; Roma, S.; Salluce, N. Adv. Funct. Mater. 2006, $16,195$. for most optical applications that use such a strategy is increasing the efficiency of the PET process, which is strongly influenced by many factors, including the donor and acceptor abilities, ${ }^{6}$ the $\mathrm{D}-\mathrm{A}$ distance, ${ }^{7}$ and the relative alignment of the energy levels of the donor and acceptor units. ${ }^{8}$ Manipulating these factors may lead to the development of functional molecules based on reversible control of the PET "on" and "off" states. When the donor and acceptor are bound through a rigid $\sigma$-spacer, the degree of throughspace electron hopping is affected mainly by the $\mathrm{D}-\mathrm{A}$

(4) (a) Velusamy, M.; Justin Thomas, K. R.; Lin, J.-T.; Hsu, Y.-C.; Ho, K.-C. Org. Lett. 2005, 7, 1899. (b) Sautter, A.; Kükrer Kaletas, B.; Schmid, D. G.; Dobrawa, R.; Zimine, M.; Jung, G.; van Stokkum, Ivo H. M.; De Cola, L.; Williams, R. M.; Würthner, F. J. Am. Chem. Soc. 2005, 127, 6719.

(5) (a) Lemaître, N.; Attias, A.-J.; Ledoux, I.; Zyss, J. Chem. Mater. 2001, 13, 1420. (b) Ishow, E.; Bellaïche, C.; Bouteiller, L.; Nakatani, K. Delaire, J. A. J. Am. Chem. Soc. 2003, 125, 15744

(6) Chow, T.-J.; Chiu, N.-R.; Chen, H.-C.; Chen, C.-Y.; Yu, W.-S.; Cheng, Y.-M.; Cheng, C.-C.; Chang, C.-P.; Chou, P.-T. Tetrahedron, 2003 $59,5719$.

(7) (a) Sankaran, N. B.; Nishizawa, S.; Watanabe, M.; Uchida, T.; Teramae, N. J. Mater. Chem. 2005, 15, 2755. (b) Lewis, F. D.; Wu, T.; Zhang, Y.; Letsinger, R. L.; Greenfield, S. R.; Wasielewski, M. R. Science 1997, 277, 673

(8) Valeur, B.; Leray, I. Coord. Chem. Rev. 2000, 205, 3. 
distance. The need for highly rigid linkers often makes the synthesis of $\mathrm{D}-\mathrm{A}$ systems rather challenging. On the other hand, through-bond electron transfer can occur efficiently in D-A systems in which the donor and acceptor units are separated by a $\pi$-conjugated linker; this process is also known as intramolecular charge transfer (ICT). ${ }^{9}$ Usually, the most efficient charge transfer occurs in systems containing fully conjugated chromophores, making it difficult to tailor the properties of the resulting D-A systems through independent modification of the structures of the donor and acceptor. Recently, we reported novel spiro-configured D-A systems that exhibit ultrafast and highly efficient PET behavior; these systems suggest that new possibilities exist for the design of novel PET chromophores. ${ }^{10}$ In these systems, the D and A units are bridged in an orthogonal manner, which affords the flexibility of fine-tuning their physical properties independently. In this paper, we report the synthesis and preliminary studies into sensor applications of novel spirobridged D-A chromophores in which the 4,5-diazafluorene (DAF) unit was adopted as the electron acceptor and triarylamines were used as electron donors. DAF derivatives exhibit high electron affinity, which makes them suitable for use as electron acceptors. ${ }^{11}$ In addition, because of their limited conformational flexibility, DAF units have enhanced chelating ability toward transition metal ions relative to that of 2,2'-bipyridine (bpy), which finds widespread use as a bidentate ligand. ${ }^{12}$ Upon their coordination to metal ions, the properties of DAF units are perturbed significantly, leading to alter their subsequent PET behavior; this phenomenon allows the new PET chromophores to be used as metal ion sensors.

Scheme 1 depicts the synthetic pathways we used to prepare some new spiro-bridged $\mathrm{D}-\mathrm{A}$ systems. The key intermediate, 4,5-diaza-2' $7^{\prime}$-dibromo-9,9'-spirobifluorene, was synthesized in $85 \%$ yield according to published procedures. ${ }^{11}$ Because the efficiency of the PET process can be modified by changing the nature of the donor and the distance between the donor and acceptor, we examined the effects of using carbazole and $N, N$-diphenylamine units as electron donors because they exhibit different electrondonating characteristics. Thus, we obtained the dipolar 4,5diaza-2', $7^{\prime}$-bis(carbazol-9-yl)-9, $9^{\prime}$-spirobifluorene (1) in 77\% yield through $\mathrm{Cu}$-mediated $\mathrm{C}-\mathrm{N}$ bond formation between 4,5-diaza-2', $7^{\prime}$-dibromo-9, $9^{\prime}$-spirobifluorene and an excess amount of carbazole in the presence of a catalytic amount of biphenyl-2-yldicyclohexylphosphane. Furthermore, we coupled 4,5-diaza-2', $7^{\prime}$-dibromo-9,9'-spirobifluorene with diphenylamine in the presence of a catalytic amount of $\mathrm{Pd}(\mathrm{OAc})_{2}$ and biphenyl-2-yldicyclohexylphosphane to afford

(9) Princiles of Fluorescence Spectroscopy, 2nd ed.; Lakowicz, J. R., Ed.; Kluwer Academic/Plenum Publishers: New York, 1999.

(10) (a) Wong, K.-T.; Ku, S.-Y.; Cheng, Y.-M.; Lin, X.-Y.; Hung, Y.Y.; Pu, S.-C.; Chou, P.-T.; Lee, G.-H.; Peng, S.-M. J. Org. Chem. 2006, 71, 456. (b) Chien, Y.-Y.; Wong, K.-T.; Chou, P.-T.; Cheng, Y.-M. Chem. Commun. 2002, 2874.

(11) (a) Wong, K.-T.; Chen, R.-T.; Fang, F.-C.; Wu C.-C.; Lin, Y.-T. Org. Lett. 2005, 7, 1979. (b) Ono, K.; Yanase, T.; Ohkita, M.; Saito, K.; Matsushita, Y.; Naka, S.; Okada, H.; Onnagawa, H. Chem. Lett. 2004, 33, 276.

(12) Tsierkezos, N. G.; Diefenbach, M.; Roithová, J.; Schroder, D.; Schwarz, H. Inorg. Chem. 2005, 44, 4969.

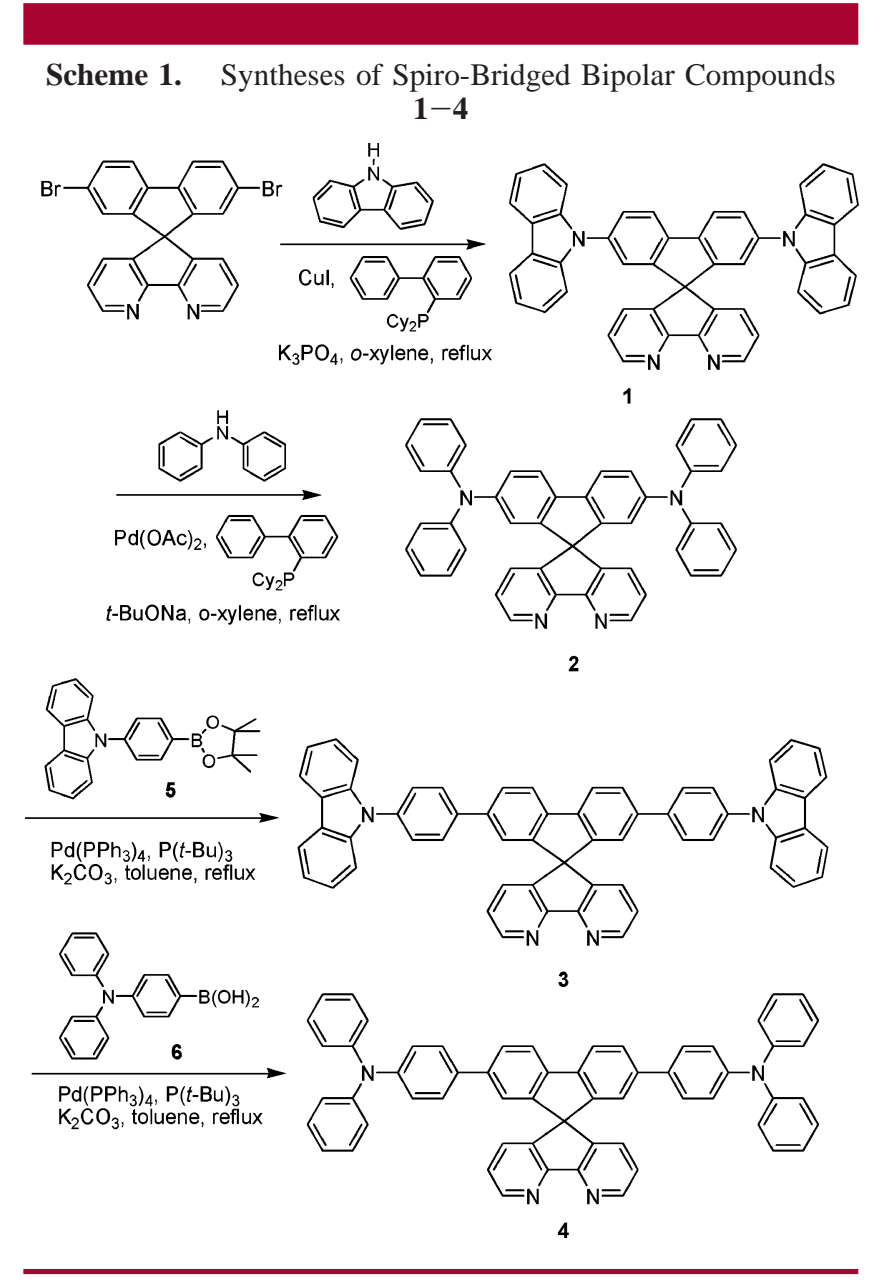

4,5-diaza-2',7'-bis( $N, N$-diphenylamino)-9, $9^{\prime}$-spirobifluorene (2) in $71 \%$ yield. The spiro-bridged D-A chromophores 3 and $\mathbf{4}$, which have relatively larger $\mathrm{D}-\mathrm{A}$ distances compared with those of $\mathbf{1}$ and $\mathbf{2}$, were synthesized in $85 \%$ and $60 \%$ isolated yields, respectively, after Suzuki couplings of 4,5-diaza-2',7'-dibromo-9,9'-spirobifluorene with the carbazole boronic ester $5^{13}$ and 4 -(diphenylamino)phenylboronic acid $(\mathbf{6}),{ }^{14}$ respectively, in the presence of a catalytic amount of $\mathrm{Pd}\left(\mathrm{PPh}_{3}\right)_{4}$ and $\mathrm{P}(t-\mathrm{Bu})_{3}$.

Figure 1 depicts the absorption and photoluminescence spectra of the spiro-bridged D-A chromophores 1-4 in $\mathrm{CH}_{2} \mathrm{Cl}_{2}$. The absorption maxima of the spiro-bridged bipolar $\mathrm{D}-\mathrm{A}$ systems possessing the same electron donor moieties (e.g., the biscarbazoles $\mathbf{1}$ and $\mathbf{3}$ ) were similar. The absorption maximum of $\mathbf{2}$ was red-shifted relative to that of $\mathbf{1}$, presumably because of the greater $\pi$-conjugation of the donor branch possessing the diphenylamino substituents. We ascribe the absorption maxima to the most prominent $\pi \rightarrow \pi^{*}$ transition of each donor branch. The absorption onsets of $\mathbf{3}$ and 4 were slightly red-shifted relative to those of their counterparts $\mathbf{1}$ and $\mathbf{2}$, respectively, presumably because of the improved $\pi$-conjugation that resulted from embedding

(13) Fushun, L.; Takashi, K.; Hiroyuki, N.; Junji, K. J. Polym. Sci., Part A: Polym. Chem. 2005, 43, 5765.

(14) Wong, K.-T.; Chien, Y.-Y.; Liao, Y.-L.; Lin, C.-C.; Chou, M.-Y.; Leung, M.-K. J. Org. Chem. 2002, 67, 1041. 


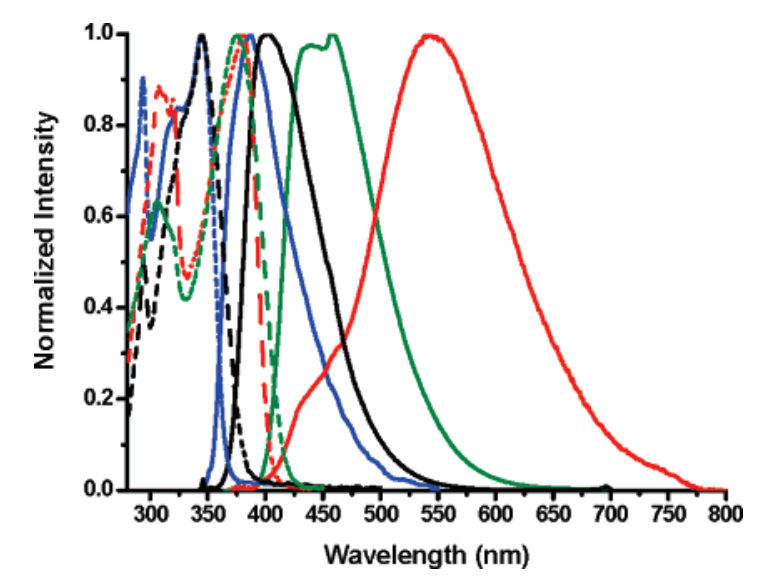

Figure 1. Normalized UV-vis absorption (dashed lines) and photoluminescence (solid lines) spectra of the bipolar compounds 1 (blue), 2 (red), 3 (black), and $\mathbf{4}$ (green) in $\mathrm{CH}_{2} \mathrm{Cl}_{2}$ at $25^{\circ} \mathrm{C}$.

two additional $p$-phenylene rings into the donor branches. The red-shifted emission maximum of $\mathbf{3}$ as compared to that of $\mathbf{1}$ agrees with a longer effective $\pi$-conjugation length of 3. The unusual red shift of the emission maximum of $\mathbf{2}$, relative to that of $\mathbf{4}$, strongly implies a mechanism different from that of typical Franck-Condon state emission in the excited state. To probe the excited-state behavior, we investigated the emission spectra of the spiro-bridged D-A systems 1-4 in different organic solvents (Figure S1, Supporting Information). The emission spectra of 1, 2, and 4 exhibit a distinct dependence on the solvent polarity, with broad emission bands that underwent bathochromic shifts upon increasing the solvent polarity. Meanwhile, their peak absorption wavelengths were effectively independent of the solvent polarity (Figure S2, Supporting Information), indicating that only limited interactions occurred between the $\mathrm{D}$ and A units in the ground state. These results are in good agreement with a mechanism involving rapid PET, leading to emissions that are dependent on the solvent polarity. Among these compounds, the bipolar chromophore 2 exhibited the most distinct PET behavior, which we attribute to the better electron-donating ability of the diphenylamino moieties and the short $\mathrm{D}-\mathrm{A}$ distance. The introduction of carbazole groups as electron-donating moieties decreased the efficiency of the PET process, as indicated by the solvent polarity only weakly affecting the emission behavior of the bipolar chromophores $\mathbf{1}$ and $\mathbf{3}$. It is evident from these results that the electronic characteristics of the donor and the $\mathrm{D}-\mathrm{A}$ distance can be used to modulate the emission maxima and PET efficiency in these systems.

Next, we used cyclic voltammetry (CV) to probe the electrochemical properties of the bipolar compounds $\mathbf{1}, \mathbf{2}$, and 4 (Figure 2). Compound 1 exhibited an irreversible oxidation during the first CV scan. Multiple CV scans of the same sample between 0 and $1.5 \mathrm{~V}$ led to the appearance (Figure S-3, Supporting Information) of new oxidation waves at ca. $1.1 \mathrm{~V}$ with successively increasing current; this phenomenon is characteristic of the electropolymerization

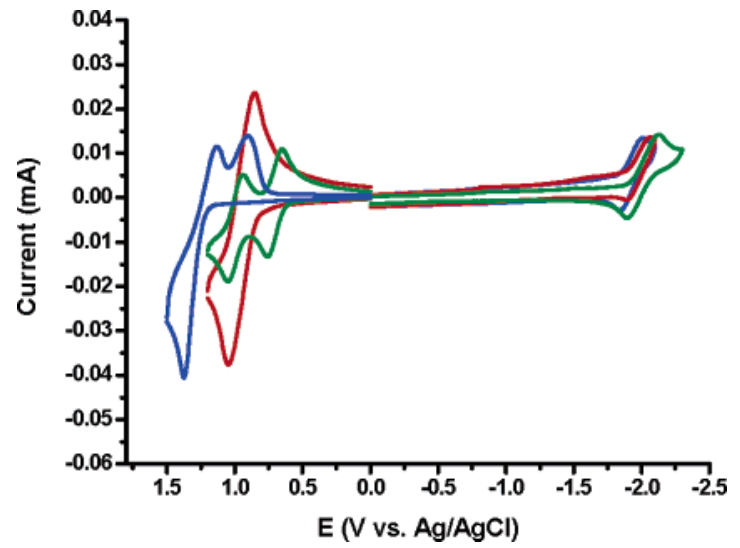

Figure 2. Cyclic voltammograms of $\mathbf{1}$ (blue), 2 (green), and $\mathbf{4}$ (red); $0.1 \mathrm{M}$ TBAP (reduction) in THF and $0.1 \mathrm{M} \mathrm{TBAPF} 6$ (oxidation) in $\mathrm{CH}_{2} \mathrm{Cl}_{2}$ were used as supporting electrolytes. A glassy carbon electrode was used as the working electrode; scan rate 100 $\mathrm{mV} / \mathrm{s}$. The $\mathrm{CV}$ of $\mathbf{3}$ cannot be obtained because of its low solubility.

of carbazoles occurring at the C3 and C6 positions. ${ }^{15}$ Two reversible oxidations at potentials of 0.71 and $0.99 \mathrm{~V}$ (vs $\mathrm{Ag} / \mathrm{AgCl}$ ) occurred for compound 2, whereas only one reversible oxidation (at $0.95 \mathrm{~V}$ ) occurred for compound 4 . The low first oxidation potential of $\mathbf{2}$, relative to that of $\mathbf{4}$, is due to significant coulombic repulsion between the two nitrogen atoms of the donor unit. The pronounced potential difference $(\Delta E=0.28 \mathrm{~V})$ between the first and second oxidations of 2 indicates that efficient resonance delocalization of the radical cation occurs in the donor branch. The comproportionation constant $\left(K_{\mathrm{c}}\right)^{16}$ can be calculated to be $5.4 \times 10^{4}$, which refers to a class II system. The two diphenylamino groups of $\mathbf{4}$ are relatively remote, resulting in their poor electronic communication and independent oxidation at a relatively higher potential. The bipolar compounds 1, 2, and 4 each underwent one reversible reduction at a reduction potential close to that of 4,5-diaza$9,9^{\prime}$-spirobifluorene; thus, we unambiguously assign the reduction site as being located on the acceptor branch. Among these three systems, compound $\mathbf{2}$ exhibited the highest reduction potential, possibly because of a weak electronic induction effect of the strongly electron-donating diphenylamino moieties on the 4,5-diazafluorene unit.

Alignment of donor and acceptor energy levels is crucial for efficient PET. The manipulation of energy levels by perturbing the electronic properties of the donor and acceptor units facilitates switching of the PET process between its "on" and "off" states. Using this strategy, PET chromophores may significantly respond to agents that alter their electronic properties of the D-A pair. Because of the strong electrondonating ability of diphenylamino group, the good electronaccepting ability of its 4,5-diazafluorene unit, and its short $\mathrm{D}-\mathrm{A}$ distance, compound $\mathbf{2}$ undergoes an efficient PET

(15) Hosseini, S. H.; Entezami, A. A. J. Appl. Polym. Sci. 2003, 90, 63. (16) (a) Bott, A. W. Curr. Sep. 1997, 16, 61. Ito, T.; Hamaguchi, T.; Nagino, H.; Yamaguchi, T.; Kido, H.; Zavarine, I. S.; Richmond, T.; Washington, J.; Kubiak C. P. J. Am. Chem. Soc. 1999, 121, 4625. 
reaction. Taking advantage of the good chelating ability of the 4,5-diazafluorene (acceptor) moiety toward metal ions, we performed a preliminarily examination of the effectiveness of modulating the PET process through metal ion complexation. Figure 3 displays the emission spectra of 2

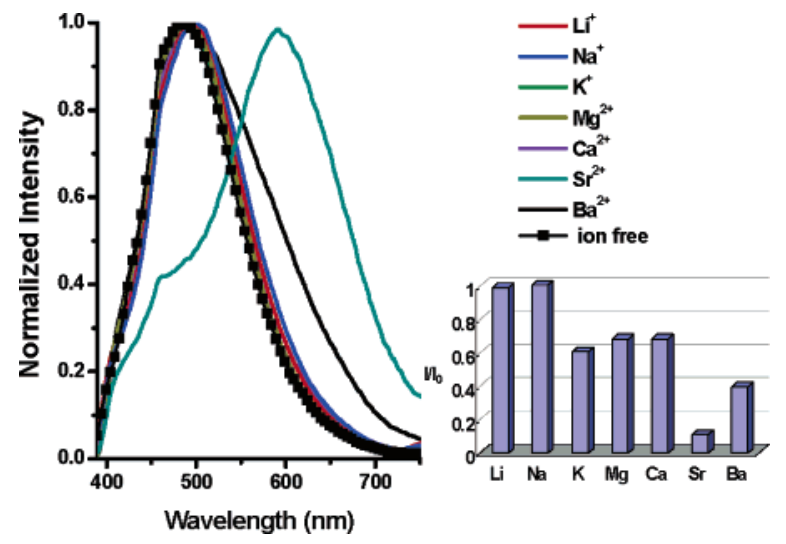

Figure 3. Normalized fluorescence spectra of $2(50 \mu \mathrm{M})$ in the presence of IA and IIA metal ions $(500 \mu \mathrm{M})$ upon excitation at $380 \mathrm{~nm}$ in THF. Inset: fluorescence responses $\left(I / I_{0}\right)$ of $\mathbf{2}$ at emission maxima after adding IA and IIA metal ions; $I_{0}$ is referred to the fluorescence intensity of ion-free state.

that we obtained in the presence of an excess amount of group IA and IIA metal ions.

Of those metal ions, only $\mathrm{Sr}^{2+}$ led to a significant red shift of the emission maximum (from 485 to $590 \mathrm{~nm}$ ) and a high degree of photoluminescence quenching. The longerwavelength emission and low photoluminescence efficiency are highly indicative of a more efficient PET process. Figure 4 displays the emission spectra of $\mathbf{2}$ obtained upon adding excess amounts of heavy metal ions. It is obvious that the presence of $\mathrm{Mn}^{2+}$ ions had a negligible effect on the emission wavelength of $\mathbf{2}$. We detected a distinct dual emission after adding $\mathrm{Zn}^{2+}$ to compound $\mathbf{2}$. The presence of $\mathrm{Ni}^{2+}, \mathrm{Cu}^{2+}$, $\mathrm{Cd}^{2+}, \mathrm{Hg}^{2+}$, and $\mathrm{Pb}^{2+}$ ions all led to substantial quenching of the photoluminescence of $\mathbf{2}$, resulting in weak residual emissions from the donor branch. These preliminary results demonstrate that the photo response of the spiro-bridged bipolar chromophore $\mathbf{2}$ is affected dramatically by the presence of metal ions that alter the electronic properties of the 4,5-diazafluorene unit. Further analysis of the structures of the metal complexes of $\mathbf{2}$ should allow us to clearly understand how the complexation event affects the PET mechanism.

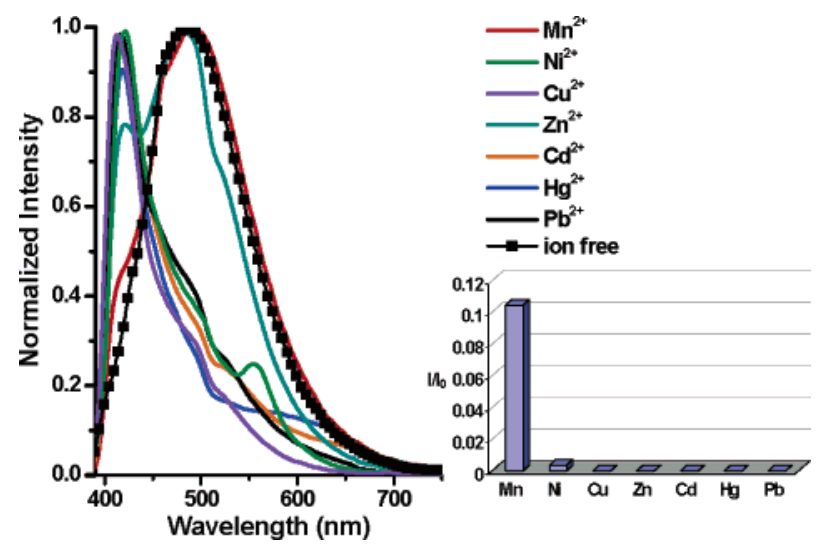

Figure 4. Normalized fluorescence spectra of $2(50 \mu \mathrm{M})$ recorded in the presence of heavy metal ions $(500 \mu \mathrm{M})$ upon excitation at $380 \mathrm{~nm}$ in THF. Inset: fluorescence responses $\left(I / I_{0}\right)$ of $\mathbf{2}$ at emission maxima after adding heavy metal ions; $I_{0}$ is referred to the fluorescence intensity of ion free state.

In summary, we have synthesized a series of novel spirobridged bipolar chromophores incorporating 4,5-diazafluorene units as electron acceptors and aryl amino groups as electron donors. The perpendicular arrangement of the donor and acceptor limits the degree of $\mathrm{D}-\mathrm{A}$ interaction in the ground state and allows efficient PET to occur in the excited state. The efficiency of this PET process was modulated by altering the $\mathrm{D}-\mathrm{A}$ distance and the electronic characteristics of the donor moiety. The bipolar molecule 2 exhibits profoundly different PET behavior in its complexed and uncomplexed states, suggesting a potential for sensing metal ions. In particular, the complexation of 2 with $\mathrm{Sr}^{2+}$ ions led to a significant change in the emission wavelength. The stoichiometric ratios and structures of the resulting complexes are under investigation and will be reported in due course.

Acknowledgment. This study was supported financially by the National Science Council of Taiwan.

Supporting Information Available: Detailed experimental procedures for the synthesis and spectroscopic characterization of new compounds; emission and absorption spectra of 1-4 in various solvents; multiple scans cyclic voltammogram of $\mathbf{1}$. This material is available free of charge via the Internet at http://pubs.acs.org.

OL061227N 